\title{
La Unión General de Trabajadores (UGT) y la Confederación Fran- cesa Democrática del Trabajo (CFDT): nuevas ejecutivas, nuevo socio sindical en Francia (1971-1978)*
}

\author{
Bruno Vargas ${ }^{1}$ \\ Université Fédérale Toulouse Midi-Pyrénées / INU Champollion \\ UMR FRAMESPA - (UT2J-CNRS)
}

RESUMEN: La opción sindical defendida por la Unión General de Trabajadores (UGT) a lo largo del exilio consistió en privilegiar el trabajo en las grandes instituciones sindicales del mundo occidental y mantener una presencia casi testimonial en España. Esta elección pudo representar un serio obstáculo a la muerte del dictador, en su lucha con Comisiones Obreras (CCOO) y sobre todo la Unión Sindical Obrera (USO) para recuperar la representatividad de los trabajadores españoles, por actuar esta última en el mismo campo ideológico.

A partir de un conjunto de fuentes impresas y fondos de archivo, custodiados en la Fundación Francisco Largo Caballero, se examinan las relaciones que la UGT entabló con la Confederación Francesa Democrática del Trabajo (CFDT). UGT intentaba, en el terreno internacional, asegurarse el liderazgo del sindicalismo socialista español en la Confederación Europea de Sindicatos (CES) y en la Confederación Internacional de las Organizaciones Sindicales Libres (CIOSL). Los nuevos dirigentes de la UGT buscaron el apoyo de la central francesa para recuperar el terreno perdido y desarmar el potencial de su gran rival tanto en el interior como en la escena internacional.

\section{PAlabras Clave: UGT; CFDT; USO; CES; CIOSL; relaciones in- ternacionales.}

* Este artículo ha sido realizado en el marco de la investigación desarrollada para el proyecto «La trayectoria internacional del sindicalismo socialista (1888-1986)», financiado por el Ministerio de Economía y Competitividad, Plan Nacional de I+D+I, HAR2013-44849-P.

Para la elaboración de este texto se ha utilizado el Archivo de la Fundación Francisco Largo Caballero, Madrid (AFFLC).

${ }^{1}$ bruno.vargas@univ-jfc.fr, ORCID iD: https://orcid.org/0000-0001-9526-4209. 
The Spanish Unión General de Trabajadores (UGT) and the French Confédération Française Démocratique du Travail (CFDT): new executives and new union partner in France (1971-1978)

ABSTRACT: The union action defended by the Unión General de Trabajadores (UGT) throughout its period of exile involved giving priority to work in the major trade union institutions of the Western world, while maintaining only a token presence in Spain. This approach could have presented a serious obstacle after the dictator's death in the struggle to reestablish representation of the Spanish workers by Comisiones Obreras (CCOO) and in particular Unión Sindical Obrera, since they shared common ideological ground.

Using a series of print sources and archival material held at the Francisco Largo Caballero Foundation, we examine the relations which the UGT established with the Confédération Française Démocratique du Travail. On the one hand, it sought to safeguard its leadership role in Spanish socialist unionism at the International Confederation of Free Trade Unions and the European Trade Union Confederation. On the other, internally, the UGT's new leaders sought support from the French trade union confederation to recover lost ground and disarm the potential of its great rival, both on the domestic and international scenes.

KEY WORDS: UGT; CFDT; USO; ETUC; ICFTU; international relations.

CÓMO CITAR ESTE ARTÍCULO/CITATION: Vargas, Bruno, «La Unión General de Trabajadores (UGT) y la Confederación Francesa Democrática del Trabajo (CFDT): nuevas ejecutivas, nuevo socio sindical en Francia (1971-1978)», Hispania, 78/259 (Madrid, 2018): 469-491. https://doi.org/10.3989/hispania.2018.013.

\section{INTRODUCCIÓN}

En la segunda mitad de los sesenta la implicación cada vez más directa de la Confederación Internacional de las Organizaciones Sindicales Libres (CIOSL) en los asuntos de la oposición obrera en España, obligó a los dirigentes de la Unión General de Trabajadores (UGT) a un constante replanteamiento de las tácticas empleadas en el interior ${ }^{2}$. Esta mayor participación tuvo repercusiones profundas, no sólo en el modelo organizativo del sindicato, sino también en su política sindical. El entonces vicesecretario general, Manuel Muiño, a finales de 1967 propuso a la dirección ugetista potenciar las federaciones de industria, los comités provinciales y otorgar más autonomía y poder centralizador a la Comisión Permanente del interior. Para llevar a cabo esas nuevas misiones, tanto las federaciones como la Permanente tendrían un

2 Estas líneas son una síntesis de un trabajo publicado bajo el título «La UGT en el exilio: 1944-1948», en ALTED, AROCA y COLLADO, 2010: 138-179. 
presupuesto mensual. Además se nombró a un enlace especial para desarrollar la información en torno a las actividades de la UGT y del PSOE en España. El periodista y escritor Juan Losada fue el encargado de representar a las ejecutivas en Madrid $^{3}$. Su tarea consistió en crear un servicio de información permanente en Madrid con corresponsales por toda la geografía española.

Sin embargo, estos cambios auspiciados por la Comisión Ejecutiva no afectaban al aparato sindical. El sector crítico en el exilio, si bien compartía los planes de reorganización de las estructuras clandestinas y los de revisión de las tácticas sindicales, también pedía una profunda renovación del equipo dirigente en el exilio y más poder decisivo para el interior. Desde este punto de vista, el desarrollo y las conclusiones del X congreso de la UGT en 1968, con pocos cambios en los puestos claves de la Comisión Ejecutiva $(\mathrm{CE})^{4}$, reforzaron en los militantes más jóvenes el sentimiento de que si nada cambiaba, la UGT corría serios peligros de verse abandonada por la CIOSL y, por lo tanto, ser absorbida por las nuevas fuerzas sindicales del interior, entre ellas la Unión Sindical Obrera (USO).

En definitiva, lo que estaba en juego era la continuidad de las organizaciones socialistas en el futuro. En el contexto de la dictadura y del exilio la pervivencia dependía en gran parte de la ayuda material de la CIOSL y de las federaciones internacionales de su órbita. Esto explica por qué las primeras luchas para la renovación se libraron en el sindicato nada más terminado el congreso de 1968. El proceso culminó con el cambio generacional y la vuelta al interior de la mayor parte de la CE tras el XI congreso de $1971^{5}$.

Casi cuatro años después del XI Congreso en el exilio, que marcó la renovación de su equipo dirigente, la UGT estaba todavía buscando la manera de reimplantarse en el tejido social español, más allá de sus dos feudos, Vizcaya y Asturias.

El indudable éxito que había representado el modelo sindical elegido por la vieja guardia ugetista a lo largo del exilio, con una fuerte movilización en las instituciones sindicales internacionales, se tornó, no obstante, insuficiente ante la escasa presencia del sindicato en el terreno de la lucha de los trabajadores españoles cuando la dictadura entraba ya en su última etapa. El rechazo de la táctica del entrismo, para no verse abocado al peligro de la recuperación

3 Entrevista a Juan Losada Martín, realizada por Juan Carlos Collado Jiménez, El Escorial, 17-03-2007, Archivo de la Fundación Francisco Largo Caballero (AFFLC), 003652-002.

4 Pascual Tomás, enfermo, fue reelegido secretario general. Su estado de salud le impidió desempeñar su función y dimitió a los pocos meses de su reelección.

5 Sobre el papel de los dirigentes o ex dirigentes de las Juventudes Socialistas ver, Entrevista a José María Martínez Cobo, realizada por Bruno Vargas, Toulouse, 3 de julio de 2007, AFFLC, 003646-001. También Entrevista a Manuel Simón Velasco, realizada por Manuela Aroca Mohedano, Madrid, 10 y 16 de noviembre y 2, 7 y 9 de diciembre de 2010, AFFLC 004209-001: 31-44. 
por parte del régimen, dejó un campo abierto, no exento de peligros, al movimiento sociopolítico de las Comisiones Obreras (CCOO) y a la USO, sindicatos que no dudaron en participar en las elecciones sindicales organizadas por la dictadura franquista.

Conscientes de que partían de una situación de clara inferioridad en este aspecto, la cúpula de la UGT, de forma muy tardía, empezó un tímido acercamiento hacia la Confederación Francesa Democrática del Trabajo (CFDT). Todo parece apuntar a que en aquellas fechas, descartado cualquier acuerdo con CCOO, el debate se centró en cómo desarmar a su más directo competidor en el campo del sindicalismo socialista: el sindicato $\mathrm{USO}^{6}$.

A partir de aquel momento, los dirigentes de la UGT consideraron que debían actuar en sintonía con el sindicato hermano de la USO en Francia, la CFDT. El camino a seguir era doble: primero entablar contactos con la central francesa para convencerla de que el viejo sindicato socialista era el más indicado para defender los intereses del sindicalismo democrático de corte socialista en España. Segundo, procurar que la confederación francesa reconsiderara su intención de defender el derecho de la USO a ingresar en la Confederación Europea de Sindicatos (CES) y luego en la CIOSL. Dos iniciativas que, en caso de no llevarse a buen puerto, dejarían a la UGT sin sus dos principales bazas en los primeros meses de la Transición.

Aquella iniciativa, como iremos estudiando en el artículo, representaba, si no una ruptura, por lo menos una inflexión notable en las relaciones que, desde 1944, la UGT mantenía con las organizaciones sindicales francesas ${ }^{7}$.

Nuestro trabajo consiste en gran medida en relatar y analizar cómo los ejecutivos de la UGT lograron en un espacio de siete años recuperar el liderazgo de la opción sindical socialista, en parte gracias a la lectura que hizo la dirección de la CFDT de la situación política y sindical en España entre 1975 y $1978^{8}$.

6 Respecto a los documentos de USO, en la actualidad no existen archivos que se puedan consultar, salvo el fondo Zufiaur que se halla en la Fundación Francisco Largo Caballero. Sin embargo, para cualquier cita referida con la acción internacional y nacional de USO, nos referiremos al libro de Manuela Aroca Mohedano (AROCA, 2017), para cuya publicación la autora consultó la documentación sin catalogar de la organización, por expresa autorización de la misma, y en la que se compendian las líneas generales de las relaciones internacionales de la USO.

7 Una buena síntesis de la actividad de la UGT en el exilio y la clandestinidad en MATEOS, 2008.

8 Respecto a la documentación de la CFDT, los documentos necesarios para llevar a cabo nuestra investigación, es decir, los de la Ejecutiva Confederal y los del Departamento de relaciones internacionales, se encuentran en buena parte en la Fundación Francisco Largo Caballero, centro documental en el que hemos realizado las consultas. 


\section{UNAS RELACIONES BAJO LA SOMBRA DEL SINDICATO HERMANO FUERZA OBRERA}

Después de la creación de la CFDT en el congreso de París de noviembre de 1964, el primer documento que, de alguna manera, inicia las relaciones entre la nueva central francesa y la UGT tiene por fecha el 4 de octubre de 1965. Por parte de ambos sindicatos existía poco interés por entablar dichas relaciones. Esa carta dirigida por André Soulat, secretario general de la Federación del Metal, solo tenía un interés protocolario tendente a dar la nueva dirección de su federación a la secretaría general de la UGT 9 .

La lectura de la correspondencia entre las diferentes federaciones de la CFDT y la secretaría general o la secretaría internacional de la UGT, a partir de esa fecha hasta mediados de los setenta, permite dejar constancia del apoyo moral y a veces financiero de la central francesa a su homóloga española. Las relaciones, sin embargo, no lograron alcanzar el grado de profundidad que existía entre el sindicato socialista español y la Confederación General del Trabajo - Fuerza Obrera (FO) ${ }^{10}$.

A lo largo del exilio, el sindicato creado por Léon Jouhaux y Robert Bothereau en 1948 se había convertido en el gran aliado y protector de la UGT en Francia. Más allá de la ayuda material que FO proporcionaba al sindicato socialista español, los principales dirigentes de la central francesa no habían dudado un solo instante en abrir las puertas de las más altas instancias sindicales internacionales para que la UGT estuviese presente como miembro permanente en la CIOSL (1949) y en la CES (1973). Una presencia que cobraría una enorme importancia durante la dictadura y en los primeros años de la Transición, para la más veterana de las centrales sindicales españolas.

FO, en 1949, fue el primer sindicato francés en crear un secretariado español, dirigido por el militante ugetista Arsenio Jimeno. No obstante, ese secretariado no logró desempeñar una labor esencial de cara a los centenares de miles de emigrantes que llegaron a Francia a partir de 1956. La debilidad del sindicato francés en los sectores de la construcción, de la agricultura o del servicio doméstico explica en parte ese encuentro fallido entre la UGT y aquella emigración económica. Por otra parte, los dirigentes de FO, al igual que la vieja guardia ugetista, no supieron captar los cambios sociales que se expresaron durante el movimiento de mayo del 68, al contrario que la CFDT, que participó activamente en el movimiento estudiantil.

9 Carta del secretario de la Fédération Générale Métallurgie de la Confédération Française Démocratique du Travail (CFDT), París, 4-11-1965, AFFLC, 000384-08.

10 Correspondencia con la Confédération Française Démocratique du Travail (CFDT), 1971, AFFLC 000384-007. 
Además, desde 1966, la CFDT y la Confederación General del Trabajo (CGT) comunista entablaron una serie de encuentros en busca de un acuerdo cuyo objetivo principal era la movilización de los trabajadores. El acuerdo firmado entre las dos centrales francesas en enero de 1966 y que, sin duda alguna, impactó en la dinámica social en los años anteriores al movimiento de Mayo de 1968, indispuso sobremanera a $\mathrm{FO}^{11}$.

Las viejas y profundas relaciones entre la cúpula de la UGT y la central de André Bergeron, y el anticomunismo heredado de la guerra civil y de la guerra fría, explican, en gran medida, ese recelo hacia la confederación. Había que mantenerse, por lo menos, a la expectativa en materia de relaciones con la CFDT.

Un buen ejemplo del recato de la UGT en profundizar en estas relaciones nos lo ofrece la duda que tuvieron los dirigentes ugetistas en contestar de forma positiva a la invitación de la CFDT a participar en su 35 Congreso de mayo de 1970. Manuel Muiño, que había sustituido a Pascual Tomás en la secretaría general, se sintió obligado a recabar la opinión del secretario general de FO André Bergeron antes de dar una respuesta. Finalmente, la ejecutiva aceptó, después de escuchar al mandatario de FO decir que «era imposible no aceptar la invitación, aunque él personalmente (sic) ten[ía] reservas respecto a la sinceridad de la invitación». Dicho de otro modo, la UGT debía cuidarse de estrechar relaciones y tenía que mantener ciertas distancias con la central dirigida por Eugène Descamps ${ }^{12}$. El miembro de la ejecutiva, Ramón Porqueras Fonfría fue, como consecuencia, el primer ejecutivo de la UGT en acudir a un congreso de la CFDT desde su creación.

Lo cierto es que antes del XI Congreso de la UGT en el exilio, celebrado en Toulouse en agosto de 1971 - que la mayoría de los estudiosos definen como el de la renovación del sindicato español-, aunque existieran ciertos contactos entre ambas organizaciones, no se habían traducido en encuentros o reuniones entre ambas ejecutivas. La exclusividad de las relaciones con FO impedía cualquier tipo de unión, por lo menos al más alto nivel.

Sin embargo, algunos militantes, después de los acontecimientos del Mayo 68 en Francia, mantenían contactos en el ámbito de las federaciones. La ejecutiva siempre frenó aquellos intentos, como lo prueba su negativa a enviar una delegación al congreso de la Federación del Metal de la CFDT en mayo de 1971, pretextando una sobrecarga de trabajo debido a las recientes huelgas en España ${ }^{13}$.

\footnotetext{
${ }^{11}$ Sobre el particular, GEORGI, 1995 y NARRITSENS, 97/6 (París, 2006): 6-11.

${ }^{12}$ Carta de Muiño a Bergeron, 25-03-1970, Respuesta telefónica de Bergeron, Toulouse, 14-04-1970, AFFLC, 000384-007.

13 Correspondencia con la Confédération Française Démocratique du Travail (CFDT, Fédération Générale Métallurgie, 1969-1972, AFFLC, 000487-019.
} 
Esa falta de proclividad hacia el sindicato francés se debía en parte a las relaciones privilegiadas que la CFDT mantenía con un probable competidor en el nuevo panorama sindical español: la USO. Estas relaciones privilegiadas, sin embargo, no habían impedido que, a través de sus federaciones, la central francesa ayudara en varias ocasiones material y económicamente a militantes ugetistas perseguidos por la dictadura ${ }^{14}$.

Las relaciones entre la USO y la CFDT habían comenzado recién creada esta última y, de hecho, se inscribían en la continuidad de las que ya había mantenido la Confederación Francesa de los Trabajadores Cristianos (CFTC), precursora de la CFDT, con el primer secretario general del nuevo sindicato español, Eugenio Royo. La primera misión de envergadura llevada a cabo por la CFDT en España tuvo lugar en diciembre de 1968. Una delegación viajó a España para conocer in situ la situación política y económica del país e interesarse, en primer lugar, por el estado de la oposición sindical clandestina. Su periplo les llevó a entrevistarse con sindicalistas de UGT, USO y CCOO en Barcelona, Madrid, Gijón y Bilbao. Por su proximidad ideológica, tanto en el aspecto del discurso como en el de la actividad sindical, USO fue la organización que mejor impresión causó a los delegados franceses. A partir de entonces, estos decidieron que ayudarían tanto material como políticamente a la USO sin por ello dejar de lado a las demás organizaciones sindicales en su lucha contra la dictadura ${ }^{15}$. Aquella decisión venía a reforzar los lazos ya existentes entre federaciones, entre otras las más destacadas, como la del Metal y la de Industrias Químicas, esta última dirigida en 1968 por el futuro secretario general de la CFDT, Edmond Maire.

Sin embargo, el cambio generacional, ocurrido en el XI Congreso de la UGT en el exilio, también se nutrió de una sensibilidad sindical diferente que entroncaba en Francia con las nuevas coordenadas políticas y sindicales «postsesentayochistas». Entre los miembros de las Juventudes Socialistas, los pocos ugetistas de la segunda generación del exilio y de la nueva emigración de los sesenta, la CFDT apareció como un sindicato socialista, anti-estalinista, que ofrecía, por lo tanto, una opción diferenciada respecto a la CGT y, al mismo tiempo, una alternativa a FO, a la que se consideraba un sindicato pro gubernamental. Por lo tanto, la CFDT se presentaba como una alternativa con nuevas preocupaciones. No puede descartarse que el discurso radicalizado y anticapitalista adoptado por la CFDT encontrara un eco favorable en las nuevas generaciones de ugetistas tanto del interior como del exilio y este discurso fue uno de los elementos que alimentó las discusiones en los congresos de la

14 Correspondencia de Tesorería con sindicatos extranjeros, Correspondencia con Confédération Française Démocratique du Travail (CFDT), 1971, AFFLC, 000407-017.

15 Correspondencia con la CFDT, carta sobre la Misión en España de la CFDT, 1968, AFFLC, 000384-007. 
UGT y del PSOE entre 1971 y 1976. Atraídos por esta nueva sensibilidad sindical que exhibía la CFDT, unos pocos de aquellos jóvenes militantes de la UGT se afiliarán a ella ya entrando en la década de los setenta ${ }^{16}$. Era un salto cualitativo, pero también moral, ya que, como hemos señalado, FO siempre había estado al lado de la UGT, tanto en Francia como en las instituciones sindicales internacionales, defendiendo su derecho a ingresar en estas, a pesar de ser un sindicato en el exilio. Manuel Simón, que fue uno de aquellos militantes que se afiliaron a la CFDT y que luego desempeñaría la dirección de la Secretaría de relaciones internacionales de la UGT (1976-1986), lo expresa de manera diáfana: «... [FO] no era la militancia que necesitábamos en aquel entonces o reclamábamos en aquel entonces» ${ }^{17}$. No fue el único en dar ese paso. En la región de Lyon hubo metalúrgicos ugetistas que también ingresaron en la federación del Metal de la CFDT. En la sección de París, un grupo de jóvenes militantes ingresaron, a su vez, en la confederación francesa. El espíritu sesentayochista se había apoderado de gran parte de la juventud y de una minoría de la clase obrera reacia a las posiciones de la CGT.

Asimismo, el caso emblemático de la empresa Lip de Besançon, con el intento por los empleados de poner en práctica el sistema de autogestión de la fábrica, tuvo un impacto enorme en la sociedad francesa ${ }^{18}$. Por último, no hay que descartar el gran esfuerzo de la CFDT en llevar una política voluntarista y novedosa de cara a la emigración que sin duda alguna le permitió granjearse simpatías en la comunidad española exiliada y en la emigración económica. De hecho, la comisión nacional de trabajadores inmigrantes de la CFDT contaba con una fuerte presencia de sindicalistas españoles. Además, uno de ellos, Paco González, era el responsable de la mano de obra inmigrante española en el secretariado nacional de los trabajadores inmigrantes del sindicato francés ${ }^{19}$.

\section{UN LENTO PERO IMPRESCINDIBLE ACERCAMIENTO HACIA LA CFDT}

El cambio de dirección en el seno de la UGT a partir de agosto de 1971 no significó un cambio radical en la política de alianza sindical con los sindicatos franceses. Como subraya acertadamente el dirigente ugetista Manuel Simón, la UGT tuvo sus problemas para establecer unas relaciones fluidas con

16 Entrevista a Manuel Simón Velasco, realizada por Manuela Aroca Mohedano, Madrid, 10 y 16 de noviembre y 2, 7 y 9 de diciembre de 2010, AFFLC, 004209-001: 41-42.

17 Entrevista a Manuel Simón Velasco, realizada por Manuela Aroca Mohedano, Madrid, 10 y 16 de noviembre y 2, 7 y 9 de diciembre de 2010, AFFLC, 004209-001: 41-42.

18 GEORGI, 2003: 452-466.

19 KAHN y VARGAS, 2012: 71. 
la CFDT. El interlocutor natural de la central francesa seguía siendo la USO por razones ideológicas más que evidentes. Tal vez lo que propició una mejora en las relaciones entre ambas ejecutivas fue que, desde el principio, tanto los unos como los otros coincidieron en que solo una fusión entre la UGT y la USO podría consolidar el sindicalismo socialista frente a las potentes CCOO. Consolidar únicamente las siglas USO dentro de una estructura supranacional, entiéndase la CES, solo conduciría al fraccionamiento del sindicalismo de obediencia socialista y al reforzamiento de la hegemonía de Comisiones ${ }^{20}$. Tampoco habría que desdeñar el peso que representaba la UGT en la nueva organización sindical europea, probablemente más símbolico que efectivo. Y viene bien recordar aquí que UGT fue el único sindicato en el exilio, con algo más de dos mil afiliados, que se unió a las dieciséis confederaciones fundadoras para crear la CES.

Ello debió pesar para que los dirigentes de la CFDT accedieran a profundizar las relaciones con la UGT, aunque siguieran dudando mucho sobre el camino a seguir. Puede decirse incluso que hasta mediados de 1977, como veremos más adelante, no quisieron alterar su estrategia en el caso español, que consistía en apoyar sin fisuras a la USO y en mejorar sus relaciones con la UGT. La CFDT dejó claro en el escenario europeo su anhelo de que la USO ingresara en la CES y no tuvo reparo en apoyar la entrada de CCOO, si con ello se permitía que la USO formara parte del «mismo paquete» ${ }^{21}$.

Aquella posición de la CFDT de alguna manera obligó a los dirigentes de la UGT a buscar nuevas vías de entendimiento y a prestar un interés creciente a las relaciones entre ambas centrales. Manuel Simón, que estuvo en primera línea de estas relaciones, incluso antes de que llegara a la secretaría de relaciones internacionales, considera que aquel fue un objetivo de primer orden. Sin embargo, esto no impidió que la UGT prosiguiera con los lazos históricos que le unían con FO y que incluso hubiera algún contacto con la CGT22.

Hubo mucho pragmatismo en aquel acercamiento, más allá de las coincidencias doctrinales. La UGT necesitaba contrarrestar las excelentes relaciones entre la USO y la CFDT, que eran sindicatos gemelos en lo ideológico. Ambos propugnaban una práctica sindical de clase y de masas y reivindicaban un socialismo autogestionario sin olvidar, por supuesto, sus raíces cristianas. Para los socialistas ugetistas, el objetivo principal —y casi nos atreveríamos a decir único-, consistió en rebajar la presión internacional ejercida por la

20 Entrevista a Manuel Simón Velasco, realizada por Manuela Aroca Mohedano, Madrid, 10 y 16 de noviembre y 2, 7 y 9 de diciembre de 2010, AFFLC, 004209-001: 101.

21 Entrevista a Manuel Simón Velasco, realizada por Manuela Aroca Mohedano, Madrid, 10 y 16 de noviembre y 2, 7 y 9 de diciembre de 2010, AFFLC, 004209-001: 145.

22 Entrevista a Manuel Simón Velasco, realizada por Manuela Aroca Mohedano, Madrid, 10 y 16 de noviembre y 2, 7 y 9 de diciembre de 2010, AFFLC, 004209-001: 193. 
CFDT para que la USO ingresara en la CES y/o la CIOSL aunque el sindicato francés estuviese afiliado a la Confederación Mundial del Trabajo (CMT) ${ }^{23}$. Sin embargo, desde principios de los setenta, la central de Edmond Maire había decidido promover una línea sindical internacional que desbordara el estrecho marco de la CMT. El principal escollo, según los dirigentes, radicaba en la incapacidad de la internacional cristiana para aportar una respuesta global en la lucha contra las fuerzas capitalistas mundializadas ${ }^{24}$.

En aquellos momentos finales del franquismo y principios de la transición, era vital para la UGT en su lucha contra CCOO conservar la exclusividad del sindicato español en las dos confederaciones sindicales internacional y europea. Por lo tanto, nos encontramos ante un pragmatismo en sumo grado, pero no solo.

En efecto, la dirección renovada de la UGT, esa generación que calificamos de post-sesentayochista, sobre todo a partir del XXX congreso celebrado en Madrid en abril de 1976, encontró en el discurso de la CFDT sobre solidaridad internacional, sobre inmigración, o sobre temas como los riesgos medioambientales, todo un campo de actuación sindical nuevo que compartían en gran medida ${ }^{25}$.

Esa política de acercamiento se aceleró a principios de 1975. Unos meses antes, los acontecimientos de la Revolución de los Claveles en Portugal habían dado lugar al desembarco de varias delegaciones de las dos grandes internacionales sindicales, pero también de algunas delegaciones nacionales, entre ellas la de la CFDT. Aquello permitió al delegado de la CIOLS, el ugetista Manolo Simón, conocer y entablar relaciones con la plana mayor del sector internacional del sindicato francés: René Salanne, Pierre Evain y Jacques Chérèque entre otros. Ambas centrales coincidían en el análisis de que había que evitar a toda costa la unificación de todos los sindicatos en una confederación unitaria dominada por los comunistas. Esta misión se había malogrado en el caso portugués y, probablemente, este resultado influyó en los sindicalistas de la CFDT a la hora de abordar una posición en el caso español.

La UGT tomó la iniciativa en su deseo de estrechar sus relaciones con la CFDT, aun a riesgo de poner en entredicho los lazos históricos mantenidos con FO desde hacia tres décadas. El secretario de Organización en el exterior, Antonio García Duarte, solicitó una reunión entre los máximos representantes de ambos sindicatos. El departamento internacional de la CFDT contestó de forma positiva, dos meses después, por boca de su responsable René Salan-

${ }^{23}$ La CFDT permanecería en la CMT hasta 1979 para ingresar finalmente en la CIOSL en 1988.

24 ROCCATI, 2014: 350-352.

25 Entrevista a Manuel Simón Velasco, realizada por Manuela Aroca Mohedano, Madrid, 10 y 16 de noviembre y 2, 7 y 9 de diciembre de 2010, AFFLC, 004209-001: 193. 
ne $^{26}$. La reunión, a la que asistieron por parte española Nicolás Redondo, Pablo Castellano, Antonio García Duarte y René Salanne, Jacques Chérèque, Jean Bourhis y Pierre Evain (secretarios del departamento internacional) por la delegación francesa, tuvo lugar en París el 25 de febrero ${ }^{27}$. El breve informe redactado por la delegación española indicaba de forma nítida que tanto por parte de la UGT como de la CFDT se trataba de medir si, en un futuro próximo, los dirigentes españoles podrían llegar a acuerdos tendentes a recibir el apoyo de la central francesa en el marco de la CES para que la UGT fuera el único representante del sindicalismo socialista español ${ }^{28}$.

En la CFDT de aquel entonces quienes se ocupaban de la política internacional formaban una especie de «casta» aparte. Lo cual no significaba, como bien apunta la historiadora Claude Roccati, que no gozaran de influencia, sino más bien lo contrario ${ }^{29}$. Muy significativo, en cuanto a la importancia que las cuestiones internacionales tenían para la CFDT, resulta el que los sindicalistas que llegaban al sector internacional habían ocupado antes cargos de alta responsabilidad en el sindicato y eran considerados pesos pesados de la organización $^{30}$. Y de hecho, hubo tal vez alguna frustración en hombres como los ya citados René Salanne, Pierre Evain y Jacques Chérèque, pero también en Jean Bourhis y René Briesh, cuando constataban que su trabajo en la escala internacional se veía frenado por su adscripción a la federación internacional sindical de orientación cristiana. Eran insalvables las dificultades que la CFDT encontraba para acercarse sola y mantener relaciones de trabajo con las organizaciones afiliadas a la CIOSL. Es probable que su competidora en el ámbito francés, FO, no estuviera ajena a esa obstaculización. Desde mediados de los sesenta, los dirigentes de la CFDT estaban convencidos de que solo si se lograse constituir una fuerza sindical europea, podrían salvar el obstáculo. De ahí que sus relaciones con otros sindicatos extranjeros le otorgaran a la CFDT un reconocimiento en la escena internacional ${ }^{31}$. Los lazos con la UGT, miembro de pleno derecho de la CES como ella, pero sobre todo de la CIOSL, más allá de un posible proyecto sindical compartido, le daban ese papel de referente tan anhelado fuera del ámbito cristiano, que ocupaba, por ejemplo, un sindicato como la USO en España. Eran conscientes, sin embargo, de que esto

26 Antonio García Duarte a Edmond Maire, 2-01-75, Pierre Evain a Antonio García Duarte, 20-02-75, AFFLC, 000442-003.

27 Entrevista CFDT-UGT, París, 25-2-1975, AFFLC, 000442-003. ORTUÑO ANAYA, 2005: 162 .

28 Entrevista CFDT-UGT, París, 25-2-1975, AFFLC, 000442-003.

29 ROCATTI, 2014: 710.

30 ROCATTI, 2014: 710. A modo de ejemplo citaremos a los dos dirigentes del Metal Jacques Chérèque et Albert Mercier.

31 ROCATTI, 2014: 711. 
no bastaba ya para lograr superar la barrera de su afiliación a la internacional sindical cristiana ${ }^{32}$.

Por su parte, la ejecutiva ugetista buscó cualquier motivo para estrechar esos lazos. Se intentó relacionar sindicalistas ugetistas que trabajaban en filiales españolas de multinacionales francesas con sus homólogos de la CFDT, como es el caso de la empresa Moulinex. Los dirigentes de la central francesa accedieron, sin mayores obstáculos, a los deseos de la central socialista española $^{33}$. Los ugetistas también procuraron contrarrestar la política sindical de la USO quejándose de sus actividades antiugetistas en España y en el exterior, singularmente en París, donde la USO mantenía una oficina que gozaba del apoyo de la CFDT desde su instalación en la capital francesa a mediados de los sesenta ${ }^{34}$.

Lo cierto es que a partir de diciembre de 1975 y a lo largo de todo el año siguiente hubo una relación fluida entre la ejecutiva de la UGT y federaciones de la CFDT, entre ellas la del Metal de la central francesa ${ }^{35}$.

A finales de 1975, la ejecutiva de la UGT cursó una invitación a René Salanne para que una misión de la CFDT visitara España y pudiese darse cuenta de la implantación de la UGT. A principios de enero, el responsable del sector internacional de la central francesa daba su acuerdo, dejando bien claro, sin embargo, que esa visita se haría en paralelo con «nuestros camaradas» de la $\mathrm{USO}^{36}$.

René Salanne lo había planeado de forma minuciosa. Para evitar cualquier conflicto con los dos sindicatos socialistas españoles, cuatro militantes de la CFDT formarían dos grupos para visitar separadamente a las delegaciones de la UGT y de la USO durante su estancia en Barcelona, Murcia, Sevilla, Madrid, La Coruña, El Ferrol y Oviedo. Esta forma de proceder no respondía exactamente a la invitación de la dirección de la UGT, la cual hubiese preferido que esa visita se circunscribiera solo a los militantes ugetistas. Además, la UGT se vio forzada a aceptar que en la conferencia de prensa prevista en Madrid, solo comparecerían la USO y la CFDT.

A pesar de esos inconvenientes, aquella visita, que tuvo lugar del 28 de febrero al 6 de marzo, se presentaba como decisiva para el futuro de las relacio319-330.

32 Para un análisis del proceso de ingreso de la CFDT en la CES, ver ROCCATI, 2014:

33 Antonio García Duarte a Jacques Chérèque, 26-05-1975 y José María Benegas a Nicolás Redondo, 26-05-1975, AFFLC, 000442003.

34 Antonio García Duarte a Jacques Chérèque, 26-05-1975 y Antonio García Duarte a René Salanne, 24-12-1975, AFFLC, 000442003.

35 Correspondencia con el sindicato francés FGM-CFDT, AFFLC, 001668 022, 001668 024. Correspondencia de José Luis Daza, secretario de Relaciones Internacionales de UGTMETAL con la Fédération de la Métallurgie de la CFDT, AFFLC, 001734003.

36 René Salanne a Antonio García Duarte, 7-1-76, AFFLC, 000442-003. 
nes entre la UGT y la CFDT, especialmente para la primera, por su voluntad de seguir siendo la central hegemónica del sindicalismo socialista español en las instituciones internacionales.

En este aspecto, la UGT logró con creces sus aspiraciones. René Salanne no ocultó que los delegados de la CFDT regresaron encantados y que sacaron buenas impresiones de las numerosas reuniones que tuvieron con militantes de la UGT. A raíz de aquel viaje, el secretariado nacional del sindicato francés reiteró su ofrecimiento de un nuevo encuentro con la dirección ugetista. Es probable que a tenor del contenido de la carta y del tono empleado, la ejecutiva de la UGT pensara razonablemente que la CFDT tal vez estaba evolucionando hacia sus posiciones en relación con el porvenir del sindicalismo socialista en España ${ }^{37}$. Más aun cuando dos meses después, tal como lo habían propuesto, una delegación encabezada por René Salanne permaneció reunida durante tres días con la plana mayor de la UGT en Madrid ${ }^{38}$.

Sin embargo, parece que la CFDT tuvo sus dudas sobre la línea a seguir con la UGT. En efecto, en el mismo mes de marzo de 1976, una reunión del buró nacional de la central francesa, después de analizar el informe de la misión en España de principios de febrero, concluía que su perspectiva de futuro consistía en primer lugar en priorizar el trabajo con USO. No obstante, los dirigentes de la CFDT estaban de acuerdo en que no se debían desechar los contactos con la UGT y que, al contrario, debían mantenerse e incluso desarrollarse. Tambien, se hacía hincapié en que se debían incrementar las relaciones con el Sindicato Vasco Solidaridad de Trabajadores (STV). Pero insistían, la prioridad de las prioridades consistía en ayudar a la USO, porque ambas organizaciones compartían la misma visión de un sindicato de masas, socialista y autogestionario. Es un detalle importante que el análisis que aquellos dirigentes de la CFDT hacían del panorama sindical español les llevaba a pensar que la USO estaba destinada a desempeñar un papel fundamental en la corriente sindical socialista en España. Asimismo recordaban que esa reflexión no era de ahora y que se había traducido hasta ese momento en unas relaciones estrechas, no solo a nivel confederal, sino también a nivel de federaciones, entre ellas las más importantes con la Federación del Metal liderada por Jacques Chérèque (1971-1979) y con la de las Industrias Químicas, liderada por Edmond Maire hasta 1972. Por fin, se ponía de manifiesto que la ayuda de la CFDT había sido importante en los momentos de la clandestinidad ante les dificultades sufridas por la USO (juicios a militantes, escisión de 1970, etc.), pero también que para profundizar en la orientación y la acción común esta ayuda no podía abandonarse ahora que el país había entrado en una vía de democratización. Por lo tanto, el secretariado nacional alentaba a la mili-

37 René Salanne a Antonio Garcia Duarte, 13-2-1976, AFFLC, 000442-003.

38 René Salanne a Antonio García Duarte, 18-03-1976, AFFLC, 000442-003. 
tancia a que se volcara en este asunto y a que se conociera más y mejor a la USO en las estructuras del sindicato. Para tal cometido se distribuyó a todas las regionales, federaciones y uniones departamentales un número especial redactado por Raimon (pseudónimo de Fernando León) de la delegación exterior de la USO en París.

Finalmente, René Salanne argumentaba que lo más urgente era la ayuda material que se debía otorgar a la USO para que pudiese luchar, según decía, en condiciones iguales con la UGT y CCOO, que recibían importantes medios de sus respectivas internacionales y de los sindicatos nacionales amigos ${ }^{39}$.

La ayuda económica de la CFDT a la USO fue constante entre 1975 y 1977, como lo prueba la correspondencia entre Francisco León y el sector internacional del sindicato francés. Ahora bien, no fueron cantidades muy elevadas, ya que si nos atenemos a las demandas del responsable de la oficina exterior de la USO en París, representaron unos 70000 francos de la época ${ }^{40}$.

En ese apoyo de la CFDT a la USO, no hay que desestimar el aspecto histórico y humano. Las relaciones entre ambas organizaciones remontaban a los primeros años de la CFDT (1964) e incluso en los tiempos de la CFTC, cuando Eugenio Royo estableció contactos con la central cristiana francesa desde su tiempo de presidente internacional de las Juventudes Obreras Cristianas ${ }^{41}$. Además, lo que luego se denominó el grupo de París, tal vez de forma exagerada, como grupo estable de la USO en Francia gracias al apoyo financiero de la CFDT, reforzó aquellos sentimientos de hermandad sindical. También significativa era la amistad que el líder de los metalúrgicos Jacques Chérèque y responsable del departamento internacional a partir de 1979 profesaba a Eugenio Royo ${ }^{42}$.

\section{SE IMPONE EL PRAGMATISMO SINDICAL: LA CFDT Y EL NUEVO PANORAMA SINDICAL Y POLÍTICO ESPAÑOL}

Sin embargo, el XXX congreso de la UGT, el primero celebrado en España desde 1932, iba a cambiar el rumbo de la situación sindical en España. No tanto por las resoluciones que allí se tomaron, aunque fueran de un profundo

39 Archivo de José María Zufiaur Narvaiza, Correspondencia con la CFDT (Francia), 1960-1977, AFFLC, 000798-004.

40 Archivo de José María Zufiaur Narvaiza, Correspondencia con la CFDT (Francia), 1960-1977, AFFLC, 000798-004. Estimación elaborada por el autor a partir de dicha correspondencia.

41 Entrevista a Manuel Zaguirre Cano, realizada por Manuela Aroca Mohedano, Barcelona, 16 de abril y 10 de mayo de 2010, AFFLC, 004201-00: 15.

42 Entrevista a Manuel Zaguirre Cano, realizada por Manuela Aroca Mohedano, Barcelona, 16 de abril y 10 de mayo de 2010, AFFLC, 004201-00: 19. 
calado desde un punto de vista sindical y político ${ }^{43}$, sino por el respaldo internacional que supuso para la veterana UGT. No cabe duda de que el olfato político de Nicolás Redondo y Pablo Castellano, que apostaron por la celebración del Congreso en Madrid, en vez de Bruselas como se había previsto en un primer momento, permitió que el valor simbólico que representaba la presencia del sindicato tanto en la CES como en la CIOSL, a partir de ese acto se transformara en un valor efectivo. La presencia internacional en los salones del restaurante Biarritz, donde se desarrolló el Congreso, supuso un respaldo para la UGT que nadie — gobierno, fuerzas políticas todavía en semi clandestinidad y sobre todo las organizaciones CCOO y USO - podían obviar. A pesar de no ser autorizado, sino tolerado, el congreso contó con la presencia de los máximos dirigentes sindicales de las dieciséis confederaciones afiliadas a la CES, entre ellos su secretario general Peer Carlsen y el secretario confederal Jan Kulakowski. Ahora, a la ejecutiva de la UGT le quedaba por «hacer» sindicato. Y en ello dedicaron buena parte de su actividad hasta finales de los setenta ${ }^{44}$.

A los dirigentes de la CFDT, esta nueva situación no pudo haberle pasado desapercibida. Tanto más cuanto que la puesta en escena de la reaparición en público de la UGT, con las consecuentes repercusiones en la vida política y social en España, encontró su corolario en la gran repercusión que el evento de Madrid tuvo durante los debates del II Congreso de la CES, celebrado en Londres una semana después ${ }^{45}$. Eso explicaría, en gran parte, por qué la CFDT no rehuyó las recurrentes invitaciones por parte de la organización de Nicolás Redondo.

No obstante, el asunto de la Coordinadora de Organizaciones Sindicales (COS) enfrió las relaciones entre ambas confederaciones. A finales del año 1976, parece ser incluso que la CFDT había apostado más fuerte por la USO. En efecto, ante la situación política estancada por la que estaba pasando España, con unos sindicatos que seguían viviendo en una semi clandestinidad, sin reconocimiento legal, una delegación de la CFDT se trasladó a Madrid para reunirse con los dirigentes de la USO. En un comunicado común, ambas delegaciones expresaron su deseo de impulsar la Coordinadora, ante el desa-

43 En el campo sindical, el Congreso pedía la liquidación de la CNS y se reafirmaba por la ruptura sindical y en contra de la reforma sindical promovida por Carlos Arias Navarro y Rodolfo Martín Villa. En cuanto a su estructuración, el sindicato se definía como: 1- sindicato de clase, 2- revolucionario, 3- autónomo respecto al Estado, a la patronal y a los partidos políticos. 4- partidario de la libre afiliación o no de los trabajadores, de la libre constitución de asociaciones sindicales por estos mismos y de la libertad de acción sindical. 5- unitario dentro del respeto a la libertad sindical. 6- democrático en su funcionamiento. 7- internacionalista. UGT: XXX Congreso, 1976: 61 y ss. REDERO SAN ROMÁN, 1992: 199.

44 Sobre esta nueva etapa de UGT, VEGA GARCÍA, 2011.

45 VALDÉS DAL-RÉ, 2007: 261. 
fío planteado a los sindicatos por el inmovilismo del Gobierno español en materia de derechos sociales de los trabajadores ${ }^{46}$.

Esa posición de la CFDT, respaldando a la COS, creada el 22 de julio, como plataforma de unidad de acción sindical ${ }^{47}$, de alguna manera alejaba la posibilidad de que el sindicato francés y la UGT acercaran sus posiciones. Esta veía en la COS, como mucho, un instrumento para la unidad de acción entre las tres direcciones con un fin preciso: romper con el sindicato verti$\mathrm{cal}^{48}$. Una posición a años luz de las pretensiones de USO y CCOO, que abogaban por una transformación de la COS en un sindicato unitario, una vez desaparecida la Organización Sindical Española (OSE) ${ }^{49}$.

Por lo tanto, a finales de 1976, el fin de la UGT de que la CFDT dejara de presionar a los sindicatos afiliados a la CES para que facilitaran el ingreso de la USO estaba lejos de ser alcanzado, a pesar de sus esfuerzos para profundizar en sus relaciones con la central francesa. No hay que perder de vista que la CFDT nunca abandonó su objetivo de que la USO lograra afiliarse a la CES. Para una central no alineada, que no formaba parte de la CIOSL y «estaba sin estar» en la internacional cristiana, la CFDT en palabras de Manuel Simón, tenía una visión ecuménica de lo que debía ser el sindicalismo internacional ${ }^{50}$. A partir del momento en que una organización sindical gozaba de una representación de los trabajadores de un país democrático, no podía existir ningún impedimento para que formara parte de las instituciones sindicales supranacionales. De ahí que viera con buenos ojos la posible afiliación de la USO. Incluso, en su batalla por la constitución de una verdadera fuerza sindical europea, la CFDT argumentaba que las organizaciones comunistas de la Europa occidental deberían integrar la CES, si «aceptaban libremente contribuir de manera autónoma y responsable» ${ }^{51}$. Por ese motivo, estaba a favor del ingreso de CCOO en la CES.

1977 empezó como había terminado el año anterior en las relaciones entre la CFDT y la UGT. Si bien eran cordiales y fluidas entre los dos secretarios generales, Edmond Maire y Nicolás Redondo, no pasaban de las buenas intenciones. En el encuentro que ambos tuvieron en París el 21 de marzo, a buen seguro trataron del asunto de la CES, pero lo que se dio a conocer fue que ambas centrales decidieron incrementar su colaboración intersindical, tanto en el ámbito confederal como de sus organizaciones afiliadas ${ }^{52}$.

46 Comunicado de prensa USO-CFDT, Madrid, 14-12-1976, AFFLC, 000798-004.

47 Es reseñable apuntar que la CFDT preconizaba una unión sindical en España cuando era incapaz por lo demás de realizarla en Francia.

48 MARÍN ARCE, 9 (Madrid, 1996): 18.

49 MARÍN ARCE, 9 (Madrid, 1996): 12.

50 Entrevista a Manuel Simón Velasco, realizada por Manuela Aroca Mohedano, Madrid, 10 y 16 de noviembre y 2, 7 y 9 de diciembre de 2010, AFFLC, 004209-001: 195.

51 Citado en ROCCATI, $2014: 318$.

52 CFDT, service de presse, 22 mars 2017, AFFLC, 000798-004. 
Más preocupante debió de parecerles a los dirigentes de la UGT las palabras pronunciadas por el delegado de la CFDT, Jacques Moreau, en el primer congreso confederal de la USO, celebrado del 7 al 10 de abril en Madrid. En este discurso, redactado por el secretario nacional, René Salanne, después de recordar los lazos profundos que unían a ambos sindicatos, abogaba sin rodeos por el ingreso de la USO en la CES y en organizaciones internacionales de orientación socialista «no alignadas (sic) y autogestionario». Más enigmático resultó el párrafo siguiente en el que exclamó:

Entre estas organizaciones, la CFDT que hoy tiene contactos seriosos (sic) con muchas de ellas, piensa que las que se refieren al socialismo democrático y autogestionario en España tienen en común una responsabilidad particular ${ }^{53}$.

¿Cómo interpretar aquella frase? ¿Como una llamada a la unión entre la UGT y la USO en la acción unitaria de la COS? Esta era ya una coordinadora moribunda, a quien el secretario general de la UGT había dado por finiquitada en estos primeros días de la primavera de 1977. O bien, ¿había que ver en esa proclama una incitación a una colaboración más estrecha e incluso una llamada disfrazada a la fusión entre ambos sindicatos?

Esa indecisión terminó al final de la primavera. ¿Qué ocurrió para que la CFDT finalmente optara por aceptar que la UGT representaba la única solución viable en España para representar al sindicalismo socialista y decidiera, en definitiva, apoyar la fracción mayoritaria de USO en su decisión de fusionarse con la UGT?

Dos acontecimientos precipitaron esa toma de decisión. El primero ocurrió en el campo político. Los resultados de las primeras elecciones legislativas dieron un claro vencedor en la izquierda. El PSOE con sus 118 diputados y sus más de 5 millones de votantes se afirmaba como el gran partido de la izquierda española, muy por delante del PCE. Debido a los lazos de hermandad entre el PSOE y la UGT, esa victoria política tendría obligatoriamente repercusiones a nivel sindical.

Los dirigentes de la CFDT tenían alguna experiencia al respecto. Muchos de ellos habían participado activamente en la fundación en 1960 del Partido Socialista Unificado (PSU). Su proximidad al dirigente socialista francés, Michel Rocard, y a su organización política, les habían permitido entablar relaciones con el nuevo partido socialista de François Mitterrand, cuando aquel ingresó con gran parte de los militantes de su partido en el Partido Socialista Francés (PS) en 1974. No cabe duda de que la dirección de la CFDT supo aprovechar esta proximidad a los rocardistas. Roto el programa común

53 Intervención de Jacques Moreau, miembro de la comisión ejecutiva de la CFDT, I Congreso USO, Madrid, abril 1977, AFFLC, 000798-004. 
entre los partidos de la izquierda francesa en septiembre de 1977, la CFDT se aproximó aún más a la corriente rocardista. Esa decisión le permitió empezar el largo recorrido que llevaría a la CFDT a sustituir a FO como sindicato enlace de los futuros Gobiernos socialistas, desde François Mitterrand hasta François Hollande, pasando por los gabinetes de Lionel Jospin.

Desde esta perspectiva se entiende mejor que dirigentes como Edmond Maire, René Salanne e incluso, con algunos matices Jacques Chérèque, alentaran el acercamiento del secretario general de la USO José Maria Zufiaur y de sus partidarios a la UGT. Y desde luego, la mayoría de los dirigentes de la USO había llegado a la misma conclusión. Pronto, el sector afín al secretario general de la USO entabló conversaciones con la ejecutiva de la UGT. El veterano dirigente Eugenio Royo se reunió con Luis Gómez Llorente, hombre próximo a Nicolás Redondo, con vistas a lograr la unidad del sindicalismo socialista ${ }^{54}$. Entre julio y octubre, fecha del Congreso extraordinario de la USO, se produjo la escisión en el sindicato, que tuvo dos consecuencias inmediatas. La primera, redujo la visibilidad y la capacidad de acción de la USO, unos pocos meses antes de las elecciones sindicales. La segunda, en el plano internacional, alejó la posibilidad de optar a un ingreso en la CES.

Por otra parte, ese desgarro en las filas de la organización mermó la capacidad negociadora de José María Zufiaur con los dirigentes de UGT. A partir de ese momento, no se trataba de elaborar candidaturas conjuntas, ni de fusión entre iguales, sino de negociar el encaje de una fracción de la USO en la UGT. Este proceso acelerado de fusión se concretó en el congreso extraordinario del 18 de diciembre. La presencia masiva de delegados internacionales avalaba la estrategia emprendida por la dirección ugetista desde 1975. En tres años, la vieja central socialista había recuperado su espacio sindical en España y consolidado su presencia internacional.

En cuanto a la CFDT, al final se impuso el pragmatismo sindical y no tuvo más remedio que adaptar su discurso y su práctica, en función del nuevo panorama politico español. Bien es verdad que vio cumplido, de alguna manera, su anhelo de que ambas centrales llegaran a una unidad de acción. Pero esta victoria moral, sin embargo, no podía ocultar una derrota en el plano del proyecto sindical. La integración de unos centenares de cuadros de la USO en la UGT a finales de 1977 no supuso una refundación generacional, política o ideológica de ésta, como subraya Abdón Mateos ${ }^{55}$. Pronto, las ideas de autogestión y autonomía sindical, aunque fueran debatidas en el Congreso de la UGT de mayo de 1978, pasaron al baúl de los olvidos. Hubo que esperar la crisis entre el PSOE y la UGT a partir de mediados de los ochenta para que la segunda se impusiera en la agenda del sindicato socialista.

\footnotetext{
54 MATEOS, 2002: 274-275.

55 MATEOS, 2002: 276.
} 


\section{CONCLUSIONES}

Al fin y al cabo, tanto la CFDT como la UGT vieron cumplidas sus expectativas. Con la fusión, una parte importante de los cuadros y de la militancia de la USO pudieron beneficiarse del paraguas no solo de la CES sino también de la CIOSL. Por su parte la UGT veía alejarse el peligro de su representación internacional y de cara a las próximas elecciones pudo afianzarse como representante de la corriente sindical socialista.

El resultado de las primeras elecciones sindicales, celebradadas en 1978, reforzó a CCOO, que obtuvo más de un tercio de los delegados, seguida por la UGT con el $21,7 \%$. Con todo, la UGT había sorteado el peligro que representaba su escasa representación salvo en Vizcaya y Asturias a la salida de la dictadura, recuperando en poco más de dos años el tiempo perdido respecto a CCOO y USO. Por otra parte, también había ganado la batalla de las internacionales sindicales como única representante del sindicalismo socialista en España, aunque en este aspecto, tampoco hay que olvidarse de la labor sindical y política incansable llevada a cabo en el exilio por los dirigentes de la UGT desde 1948.

El proyecto seguido por los dirigentes de la UGT, que puede calificarse de supervivencia para su confederación, supuso probablemente una merma de la representación sindical en España. En efecto, el modelo sindical defendido por la USO - autonomía sindical, práctica sindical de clase y de masas, socialismo autogestionario- desapareció en gran medida con la fusión entre ambos sindicatos en 1977. Por otro lado, cabe preguntarse si en el proceso de la Transición, una etapa eminentemente política en el que el aspecto sindical fue relegado, por lo menos hasta 1981, a un segundo plano, existía un espacio para dos proyectos socialistas frente a CCOO. Probablemente la respuesta se encuentre en las trayectorias que siguieron, a partir de 1978, la central dirigida por Nicolás Redondo y la USO de Manuel Zaguirre.

No cabe duda de que la UGT encontró un aliado insospechado, por lo menos hasta 1976, en la CFDT. La voluntad de la Confederación francesa de entablar relaciones bilaterales con los sindicatos europeos que tenían cierta presencia en el sindicalismo internacional, desempeñó un papel fundamental a la hora de acercar posiciones con la UGT. De alguna manera, al igual que la UGT, la CFDT también buscaba una vía para existir en el mundo sindical europeo e internacional. Las palabras de René Salanne, alma mater de la política internacional del sindicato francés en la década de los setenta, recordadas por Roccati en su trabajo de tesis doctoral, ilustran lo que ocurrió con el sindicato socialista:

En Europa queríamos ensanchar nuestras relaciones, lo que significa que, durante todo mi periodo, dimos la vuelta [...] a casi todas las organizaciones europeas 
[...]. Eso significa que se discute, se ve, se crea en un momento dado una reunión, se organiza un contacto, el sector internacional y el equivalente del otro lado ${ }^{56}$.

En otras palabras, la UGT ofrecía a la CFDT lo que la USO no podía, ni de lejos, aportarle: una base sólida en el sindicalismo occidental mediante su afiliación a la CIOSL. No hay que olvidar que el sindicato de Edmond Maire debía librar una ardua batalla para poder existir en el fracturado panorama sindical en Francia, donde predominaban la CGT y FO. Alejado de su internacional, integrada en la CES, después de arduas negociaciones, en mayo de 197457 , no se encontraba en posición de fuerza para defender su proyecto sindical.

¿Eran conscientes de ello los dirigentes de la UGT? A raíz de nuestra investigación, se puede razonablemente pensar que, solo a partir de 1977, empezaron a vislumbrarlo. Sin duda alguna, hubo una confluencia de intereses que facilitó el acercamiento entre ambos sindicatos a costa de la USO.

Probablemente fueron las consecuencias políticas de los comicios de junio de 1977, las que más impacto tuvieron en esa relación. La irrupción del PSOE, como fuerza alternativa a las derechas de la España posdictatorial, tras las elecciones legislativas, precipitó los acontecimientos. La hipoteca que representaba la USO en el devenir sindical de la UGT, encontró su desenlace en la victoria política del PSOE. Aceleró la desactivación del peligro sindical que representaba la USO para la central de Nicolás Redondo. Este asunto, sin negar las capacidades de finos estrategas de los principales dirigentes de UGT, se resolvió de alguna manera con la ayuda inesperada del partido hermano. Tal vez aquello entraba en la lógica de una transición que tuvo componentes esencialmente políticos.

De hecho, el congreso de la fusión entre los dos sindicatos socialistas españoles fue recibido con cierto alivio en las filas de la confederación francesa. Los cuadros de la CFDT se felicitaron de la fusión entre la UGT y los seguidores de José María Zufiaur. Adoptaron, incluso, una actitud, si no de rechazo, por lo menos de franco alejamiento, ante la posición de parte de la USO de seguir adelante con su proyecto sindical autónomo. Desde entonces, las relaciones con el otrora sindicato hermano, liderado a partir de 1978 por Ma-

56 Citado en ROCCATI, 2014: 320.

57 El congreso fundacional de la CES se celebró en febrero de 1973 y reunía a 16 organizaciones, entre ellas la UGT, siendo Fuerza Obrera la única central francesa representada. Hubo que esperar hasta mayo del 74 para que los sindicatos cristianos o de origen cristiano pudiesen ingresar en la confederación europea. En cuanto a los sindicatos de sensibilidad comunista, únicamente ingresó la CGIL italiana en 1976. La incorporación de las otras tres grandes confederaciones del sur de Europa, CGT, CCOO y CGTP, no se produciría hasta la década de los noventa, cuando, otro acontecimiento político - la caída del Muro de Berlín-, permitió convertir a la CES en la casa común de casi todas las organizaciones sindicales de Europa. 
nuel Zaguirre, se tornaron esporádicas ${ }^{58}$. Con el tiempo, la CFDT se convertiría, junto a FO, en el principal interlocutor de la UGT en las relaciones sindicales hispanofrancesas.

En definitiva, a principios de los ochenta, la UGT, y en ello siguió los pasos del PSOE en el campo político, se hallaba solidamente implantada en España. Había logrado un estatus que le permitió superar el hermanamiento, tan importante durante toda la dictadura y en los primeros años de la Transición, de los sindicatos franceses FO y CFDT.

\section{BibliOgRAFIA}

Alted, Alicia, Aroca, Manuela y Collado, Juan Carlos (dirs.), El sindicalismo socialista español. Aproximación oral a la historia de UGT (1931-1975), Madrid, Fundación Francisco Largo Caballero, 2010.

Andolfatto, Dominique (dir.), Les syndicats en France, Paris, La Documentation française, 2004.

Andolfatto, Dominique y Labbé, Dominique, Histoire des syndicats. 1906-2006, Paris, Seuil, 2006.

Aroca Mohedano, Manuela, Internacionalismo en la historia reciente de la UGT (1971-1986). Del franquismo a la estabilización de la democracia, Madrid, CINCA-FFLC, 2011.

Aroca Mohedano, Manuela, «La Unión Sindical Obrera: del nacimiento del nuevo movimiento obrero durante el franquismo a la búsqueda de espacios sindicales en la transición», Historia del Presente, 18 (Madrid, 2011): 113-132.

Aroca Mohedano, Manuela, El papel del sindicalismo en la transición. La confluencia del sindicalismo socialista: fusión USO-UGT, Madrid, Cinca, 2017.

Battais, Louisette, Guide bibliographique. CFTC-CFDT 1884-1987, Paris, IRES, 1988.

Beneyto Calatayud, Pere J., Afiliación sindical en Europa: modelos y estrategias, vol. 1, Alzira, Germania, 2004.

Bornard, Jean, De la CSIC à la CMT. 75 ans de syndicalisme international, Paris, CFTC, 1995.

Chassaigne, Philippe, Les années 1970. Fin d'un monde et origine de notre modernité, Paris, Armand Colin, 2008.

Defaud, Nicolas, La CFDT (1968-1995). De l'autogestion au syndicalisme de proposition, Paris, Les Presses de Sciences-Po, 2009.

58 Según Manuel Zaguirre, ambos sindicatos estuvieron 30 años sin tener relacion al más alto nivel, hasta el IX congreso de la USO de diciembre de 2009 en que una delegación de la CFDT volvió a asistir por primera vez desde el I congreso de abril de 1977. Entrevista a Manuel Zaguirre Cano, realizada por Manuela Aroca Mohedano, Barcelona, 16 de abril y 10 de mayo de 2010, AFFLC, 004201-00: 111. 
Dolvik, Jon Éric, L'émergence d'une île? La CES, le dialogue social et l'européanisation des syndicats dans les années 1990, Bruxelles, Institut syndical européen, 1998.

Dreyfus-Armand, Geneviève, Frank, Robert, Lévy, Marie-Françoise y ZancariniFournel, Michèle, Les années 68. Le temps de la contestation, Paris, Editions Complexe, 2008.

Gabaglio, Emilio, Qu'est-ce que la CES ?, Paris, L'Archipel, 2002.

Georgi, Frank, L'invention de la CFDT 1957-1970, Paris, Éditions de l'Atelier, 1995.

Georgi, Franck (dir.), Autogestion, la dernière utopie, Paris, Publications de la Sorbonne, 2003.

Gillespie, Richard, Historia del Partido Socialista Obrero Español, Madrid, Alianza Universidad, 1991.

Gracia García, Jordi, Estado y cultura. El despertar de una conciencia crítica bajo el franquismo (1940-1962), Toulouse, Presses Universitaires du Mirail, 1996.

Guindal, Mariano y Serrano, Rodolfo, La otra Transición. Nicolás Redondo: el sindicalismo socialista, Madrid, Unión Editorial, 1986.

Hamon, Hervé y Rotman, Patrick, La deuxième gauche, histoire intellectuelle et politique de la CFDT, Paris, Seuil, 1984 ( $2^{\mathrm{e}}$ ed.).

Juliá, Santos, Los socialistas en la política española, 1789-1982, Madrid, Taurus, 1996.

Kahn, David y Vargas, Bruno, «Aproximación a la militancia sindical de los españoles en Francia. Caso de la UGT y de la CFDT (1956-1973)», en Manuela Aroca Mohedano (dir.), Presencia y activismo de los españoles en las organizaciones sindicales europeas, 1960-1994, Madrid, Fundación Francisco Largo Caballero, 2011: 34-81.

Launay, Michel, Le syndicalisme en Europe, Paris, Imprimerie nationale, 1990.

Lemus, Encarnación, "Con la vista en Portugal y mirando a España: EE.UU. y el cambio político peninsular», Hispania. Revista Española de Historia, LXXII/242 (Madrid, 2012): 723-754.

Marín Arce, José María, «La Coordinadora de Organizaciones Sindicales (COS): una experiencia de unidad de acción sindical durante la transición», Espacio, tiempo y forma, Serie V. $H^{a}$ Contemporánea, 9 (Madrid, 1996): 295-313.

Martínez Cobo, Carlos y Martínez Cobo, José, La segunda renovación. Intrahistoria del PSOE, vol. IV, Barcelona, Plaza\&Janés, 1991.

Martínez Quinteiro, María Esther, La denuncia del sindicato vertical. Las relaciones entre España y la Organización Internacional del Trabajo (1969-1975), vol. II, 2a parte, Madrid, CES, 1997.

Mateos, Abdón, Exilio y clandestinidad. La reconstrucción de UGT, 1939-1977, Madrid, UNED ediciones, 2002.

Mateos, Abdón, UGT contra la dictadura franquista, 1939-1975, Madrid, Siglo XXI, 2008.

Moreno, Juan y Gabaglio, Emilio, El reto de la Europa social, 30 años de la Confederación Europea de Sindicatos, Valencia, Germania, 2006.

Mouriaux, René, Le syndicalisme en France depuis 1945, Paris, La Découverte (Repères) ed., 2013.

Narritsens, André, «L'accord d'unité d'action CGT - CFDT», Dossier Les cahiers d'histoire sociale de l'Institut CGT, 97/6 (Paris, 2006): 6-11. 
Ortuño Anaya, Pilar, Los socialistas europeos y la transición española, Madrid, Marcial Pons, 2005.

Pasture, Patrick, Pigenet, Michel y Robert, Jean-Louis (dirs.), L'apogée des syndicalismes en Europe Occidentale 1960-1985, Paris, Publications de la Sorbonne, 2005.

Ramos, Esther y Rodríguez, Jesús (dirs.), Archivo de la Unión General de Trabajadores de España en el exilio (1944-1976), Madrid, FFLC, 1995.

Redero San Román, Manuel, Estudios de Historia de la UGT, Salamanca, Universidad de Salamanca, 1992.

Rocard, Michel, Le PSU et l'avenir socialiste de la France, Paris, Ed. du Seuil, 1969.

Roccati, Claude, Un internationalisme entre discours et pratiques: la politique internationale de la CFDT (1964-1988), thèse de doctorat Histoire, Université du Havre, Français, 2014, <NNT: 2014LEHA0021>.

Soto Carmona, Álvaro, Transición y cambio en España, 1975-1996, Madrid, Alianza Editorial, 2005.

Soto Carmona, Álvaro y Aroca Mohedano, Manuela (dirs.), Combates por la democracia. Los sindicatos, de la dictadura a la democracia (1938-1994), Madrid, UAM ediciones, 2012.

UGT: XXX Congreso, Madrid, Akal editor, 1976.

Valdés Dal-Ré, Fernando (dir.), 30 años de libertad sindical, Madrid, Ed. Fundacion Francisco Largo Caballero, 2007.

Vargas, Bruno, «El movimiento socialista español en el exilio y la construcción de Europa (1946-1972)», en Adolfo de Luxan y Alonso Puerta (coords.), El socialismo español en el exilio y la construcción europea, Madrid, Fundación Indalecio Prieto, 2003: 41-62.

Vargas, Bruno, «UGT en el exilio: 1944-1968», en Alicia Alted, Manuela Aroca y Juan Carlos Collado (dirs.), El sindicalismo socialista español. Aproximación oral a la historia de UGT (1931-1975), Madrid, 2010: 138-179.

Vargas, Bruno, «Análisis de las relaciones entre los gobiernos del PSOE y la UGT (1982-1989)», en El renacer del sindicalismo socialista en España (1970-1994), Manuela Aroca Mohedano (dir.), Madrid, Sílex, 2014: 150-165.

Vega García, Rubén, Historia de la UGT. La reconstrucción del sindicalismo en democracia, 1976-1994, Madrid, Siglo XXI, 2011.

Zufiaur Narvaiza, José María, USO, Madrid, Avance, 1976.

Zufiaur Narvaiza, José María, «Los sindicatos, elecciones sindicales y acción institucional», en VV. AA., Cambio Social y Acción Sindical en España (1975-1983), Madrid, FFLC, 1983.

Zufiaur Narvaiza, José María, «El sindicalismo español en la transición y la crisis», Papeles de Economía Española, 22 (Madrid, 1985): 202-234.

Zufiaur Narvaiza, José María, «El sindicalismo español y Europa», Claridad, 12 (Madrid, otoño 2007): 57-66. 